\title{
Hybrid spectrum arrangement and interference mitigation for coexistence between LTE macrocellular and femtocell networks
}

\author{
Yong Bai ${ }^{1 *}$ and Lan Chen ${ }^{2}$
}

\begin{abstract}
When deploying two-tier LTE cellular networks with coexistence of macrocells and underlaid femtocells, the strategies of spectrum arrangement need to be investigated to efficiently utilize the scarce spectrum resource. Co-channel spectrum usage and dedicated-channel spectrum usage are two traditional strategies of spectrum arrangement. Nevertheless, there are pros and cons of them from the perspective of achievable network capacity in two-tier LTE network as they result in different amounts of available spectrum at each tier and disparate cross-tier interferences. To improve overall spectrum utilization, we propose a novel approach of spectrum arrangement, which is called hybrid spectrum arrangement, to take advantage of their merits. In our proposal, an underlaid femtocell can select its spectrum usage mode according to a criterion aiming to benefit both macrocell and femtocell in terms of achieved capacities. Consequently, the femtocells embedded in macrocell are self-organized as inner and outer femtocells, which operate in dedicated-channel spectrum usage and co-channel spectrum usage, respectively. Then, we examine distinct characteristics of cross-tier interferences in the context of hybrid spectrum arrangement and present corresponding schemes to mitigate the residual significant interferences. Analysis and system level simulation are given to validate the effectiveness of our proposed methods for two-tier LTE cellular network.
\end{abstract}

\section{Introduction}

A cellular network is a radio network made up of a number of radio cells. Each cell is served by a base station (BS) to provide radio coverage over a limited area. A macrocell has the widest range of cell size and is served by high-power BS with antennas mounted above rooftops. A femtocell has the coverage of $10-50 \mathrm{~m}$ and is served by an indoor BS to support stationary or low-mobility users at homes or in small offices. The BS of femtocell is usually user-installed with a connection to the cellular operator network through a wired broadband backhaul such as digital subscriber line (DSL). Furthermore, the cells of different sizes can be deployed in a hierarchical cell structure (HCS) to provide multi-tier network

\footnotetext{
${ }^{*}$ Correspondence: bai@hainu.edu.cn

${ }^{1}$ College of Information Science \& Technology, Hainan University, 58 Renmin Ave., Haikou, Hainan 570228, China

Full list of author information is available at the end of the article
}

connectivity [1,2]. Macrocells are deployed as one radio tier to cover wide areas, and femtocells are embedded inside macrocells as another radio tier to supply sporadic overage. This overlaying approach has benefits on capacity gain, better coverage, and reduced battery consumption of handsets [3]. In this article, we consider a two-tier 3GPP long term evolution (LTE) network made up of macrocells and underlaid femtocells. Within the overlapped coverage areas in such a two-tier system, a user equipment (UE) can access either macrocell or femtocell and can switch its access tier by performing vertical handoff. Specifically, when a femtocell is configured in the closed subscriber group (CSG) manner (also called "closed access"), only the users included in the femtocell's access control list are allowed to use the femtocell resources. On the other hand, a femtocell can also be configured in the open access manner, in which any user is allowed access to the femtocell. For simplicity in this article, a UE is called an MUE when it

\section{照 Springer}

(c) 2013 Bai and Chen; licensee Springer. This is an Open Access article distributed under the terms of the Creative Commons Attribution License (http://creativecommons.org/licenses/by/2.0), which permits unrestricted use, distribution, and reproduction in any medium, provided the original work is properly cited. 
is associated with a macrocell BS (namely eNodeB or eNB in LTE network), and it is called a HUE when it is associated with a femtocell BS (namely Home eNodeB or HeNB in LTE network).

After acquiring licensed spectrum, wireless cellular operator faces the question of how to utilize this scarce resource efficiently, especially when a two-tier cell structure is employed [4]. The first strategy is letting two tiers share the licensed spectrum such that macrocell and femtocell operate in co-channel frequency reuse, which is referred to as co-channel spectrum usage in this article. The other strategy is that the licensed spectrum is partitioned to separate portions and each tier operates in a dedicated spectrum portion. The latter strategy is referred to as dedicated-channel spectrum usage in this article. There are both pros and cons of them from the perspective of achievable network capacity. With co-channel spectrum usage, each radio tier is granted largest amount of available spectrum, whereas the cochannel frequency reuse of two tiers imposes severe cross-tier interferences and consequently is considered the highest risk deployment. On the other hand, the crosstier interference is lower with dedicated-channel spectrum usage, whereas the amount of available spectrum is reduced at each tier. According to Shannon formula, both the higher cross-tier interference (with co-channel spectrum usage) and the reduced amount of spectrum (with dedicated-channel usage) can become the capacitylimiting factor. To tackle the drawbacks of two traditional strategies of spectrum arrangement, we propose a novel strategy of spectrum arrangement, called hybrid spectrum arrangement, to take advantage of their merits. In our proposal, each HeNB can select a preferable spectrum usage mode by self-configuration. It is supposed that proper selection of spectrum usage mode can avoid whichever of the available bandwidth and the cross-tier interference becoming the capacity limiting factor. Therefore, the selection of spectrum usage mode should properly trade off the amount of available spectrum and the crosstier interference. In our proposal, a femtocell selects its spectrum usage mode according to a criterion such that both macrocell and femtocell can be benefited in terms of higher achieved capacity. Since the cross-tier interference is largely dependent on the relative locations between HeNBs and eNBs, the criterion for the decision making of a HeNB can be further translated to its relevant spatial condition, i.e., the relative location of a HeNB to its embedding eNB, where the embedding eNB is the closest eNB from the HeNB. According to a spatial threshold, the femtocells embedded in a macrocell are differentiated to inner and outer femtocells, which operate in the mode of dedicated-channel spectrum usage and the mode of co-channel spectrum usage, respectively. It turns out that the spectrum usage modes of femtocells are mixed within a macrocell. This is why we call the proposed strategy hybrid spectrum arrangement. When the strategy of hybrid spectrum arrangement is employed, the cross-tier interferences have their distinct characteristics comparing with that with two traditional strategies. For successful deployment of such a strategy in the two-tier LTE cellular network, we examine the severeness of four cross-tier interference scenarios under hybrid spectrum arrangement and propose the interference mitigation methods to alleviate the residual significant interferences among them. Finally, this article stresses that the strategy of hybrid spectrum arrangement along with the related interference mitigation methods formulate as an overall solution to improve the spectrum utilization for two-tier LTE cellular network.

The main contributions of the present article can be summarized as follows.

- First, we point out that two spectrum usage modes (i.e., co-channel spectrum usage and dedicatedchannel spectrum usage) have pros and cons from the perspective of achievable network capacity after analyzing their disparate effects on the amount of available spectrum and the cross-tier interferences.

- Then, we argue that the capability of femtocells to select their spectrum usage modes flexibly helps to improve overall spectrum utilization. Furthermore, we propose a practical criterion of spectrum usage selection for femtocells aiming to benefit both macrocell and femtocell from the perspective of achieved capacities, and we discuss that the decision making of a femtocell on its spectrum usage mode can be translated to the relevant spatial condition-the relative location of a HeNB to its embedding eNB.

- Next, we present the novel strategy of spectrum arrangement, hybrid spectrum arrangement, as a natural consequence of above proposed criterion and the enabled selection capability of femtocells. In fact, the femtocells embedded in macrocell can be self-organized to inner and outer femtocells according to their spatial conditions, and they operate in dedicated-channel spectrum usage and co-channel spectrum usage, respectively. We give detailed analysis of two-tier LTE network to decide the spatial thresholds on downlink and uplink for the decision making of femtocells.

- Moreover, we discuss the distinct characteristics of cross-tier interferences under hybrid spectrum arrangement and propose mitigation methods to address two residual severe interferences-the downlink interference from HeNB to MUE and the uplink interference from HUE to eNB. Eventually, we formulate an overall solution to improve spectrum utilization for two-tier LTE cellular network by 
combining the proposed strategy of hybrid spectrum arrangement and the related interference mitigation methods.

This article extends our previous study [5,6]. In this article we clarify the criterion for selecting the spectrum usage mode and derive the spatial thresholds on downlink and uplink in more details, and we examine the impacts of hybrid spectrum arrangement on all four cross-tier interference scenarios rather than two among them in [6]. Moreover, we formulate an overall solution to improve spectrum utilization for two-tier LTE cellular network by combining the strategy of hybrid spectrum arrangement presented in [5] and the related interference mitigation methods proposed in [6]. For performance evaluation, we conduct more simulation experiments by using the system parameters that conform with the latest 3GPP LTE specifications.

The rest of this article is organized as follows. Section 3 discusses the related study on spectrum arrangement and interference mitigation for two-tier cellular network, and underlines the differences of our study with the related studies. Section 3 describes how spectrum usage selection can help to improve spectrum utilization in two-tier LTE cellular network. Section 4 presents the strategy of hybrid spectrum arrangement and its implementation methods in two-tier LTE system. This section also gives detailed analysis to derive the spatial thresholds on downlink and uplink for the decision making of femtocells. Section 5 examines the severeness of four cross-tier interference scenarios under hybrid spectrum arrangement and presents methods to mitigate two residual significant interferences. In Section 6, we give performance evaluation of proposed methods via system-level simulation of two-tier LTE system. Section 7 concludes this article.

\section{Related study}

In the literature, there has been some related study of spectrum management in the context of two-tier cellular network. Claussen [7] studied the feasibility of co-channel operation between user-deployed femtocells and an existing macrocell network, and discussed key requirements for such operation such as auto-configuration and public access. Chandrasekhar and Andrews [8] proposed an optimum decentralized spectrum allocation policy for twotier FDMA (including OFDMA) network. Oh et al. [9] proposed a frequency planning for femtocells in cellular networks using fractional frequency reuse (FFR). Partial co-channel spectrum arrangement between macro- and femto- cells is investigated in [10]. With the configuration of partial co-channel spectrum arrangement as well, Lima et al. [11] investigated coordination mechanisms to opportunistically reuse resources without compromising ongoing transmissions on overlay macrocells. Güvenç et al. [12] presented a hybrid frequency assignment for femtocells mainly aiming to maintain the femtocell's coverage on the user's premises.

In order to cope with the cross-tier interference in twotier cellular networks, interference mitigation schemes by employing dynamic radio resource management (including resource partitioning, allocating, and scheduling), power control, handover, cognitive radio have been reported. Bharucha et al. [13] focused on mitigating downlink femto-cell to macro-cell interference through dynamic resource partitioning, in the way that HeNBs are denied access to downlink resources that are assigned to macro UEs in their vicinity. To avoid the strong interference between a femtocell and close-by macrocellassociated mobile stations, Sahin et al. [14] proposes a method that jointly utilizes the spectrum sensing results as well as scheduling information obtained from the macrocell BS. The authors in [15] proposed interference mitigation strategies that adjusts the maximum transmit power of femtocell users by open-loop or closed-loop power control to suppress the cross-tier interference at a macrocell BS. Shi et al. [16] analyzed the mechanism for generating the uplink interference scenarios and provided guidelines for interference mitigation in two-tier macro and femto co-existing UMTS networks. In [17], downlink femto-to-macro control channel interference is analyzed and interference reducing methods are proposed with co-channel femtocell deployment. A distributed femtocell power allocation scheme is proposed in [18] by exploiting limited channel information of neighboring macro MSs to effectively reduces cross-tier interference on them. Ndong et al. [19] addressed the uplink cross-tier interference problem and proposes a resilient solution to the near-far effect issue by utilizing the interfering macrocell information feedback through infrastructure network at the HeNB for interfered signal recovery of the symbols sent by the HUE. A self-organizing femtocell framework which is composed of three complementary control loops for co-channel deployment is presented in [20]. LópezPérez et al. [21] proposed an interference avoidance technique combining intracell handovers and power control in OFDMA two-tier macrocell-femtocell networks. Torregoza et al. [22] proposed a cognitive femtocell network architecture that incorporates cognitive radio and femtocells, and propose a joint power control, base station assignment, and channel assignment scheme for cognitive femtocell networks. Other relevant articles include [23-25]. Zhang et al. 2011 and Yu et al. 2011 [23,24] suggest intelligent spectrum allocation using cognitive radio for home networks and smart grid, and [25] studied a parallel spectrum sensing for balancing sensing performance and efficiency in cognitive radio networks. 
Here we underline the differences of the contributions in this article with the related works. Regarding the spectrum arrangement in two-tier cellular network, there are some similar ideas in the related study [12] as our proposed hybrid spectrum arrangement. However, the strategy of hybrid spectrum arrangement is not fully explored in two-tier LTE system and there is a lack of complete solution with such an arrangement. In [12], the threshold distance that separate the inner and outer regions was investigated in the context of $3 \mathrm{G}$ wireless system and determined based on interferencelimited coverage area (ILCA) of a femtocell. In this article, the criterion to determine the inner and outer regions is based on the achievable throughputs of two LTE tiers instead of the coverage of femtocells. Furthermore, the interference mitigation methods in the related works are mainly studied in the context of co-channel spectrum usage. For instance, the interference mitigation methods presented in $[16,19]$ were targeted for the uplink interference from a visiting MUE to near-by HeNB, which is not a significant interference scenario with hybrid spectrum arrangement. Thus, instead of investigating all the possible cross-tier interferences, we examine the cross-tier interference in the context of hybrid spectrum arrangement, identify two significant interference scenarios including the interference from HeNB to nearby MUEs and the interference from HUEs to the eNB. Then, we propose the interference mitigation methods targeted for the above two severe cross-tier interferences. As far as we know, the proposed interference mitigation methods for these two scenarios in this article have not been reported in the literature before. Finally, this article gives a more complete solution for spectrum utilization in two-tier LTE cellular network by combining the strategy of hybrid spectrum arrangement and necessary interference mitigation methods under such an arrangement.

\section{Spectrum usage selection to improve spectrum utilization in two-tier LTE cellular network}

\subsection{Comparison of spectrum arrangement strategies}

The main purpose of studying the strategies of spectrum arrangement for two-tier LTE cellular network is to increase the overall spectrum utilization, which can be measured by area spectral efficiency (ASE). The ASE of a cellular system is defined as the achievable throughput per unit area for the bandwidth available. The ASE of combined macrocells and femtocells can be measured in terms of $\mathrm{bit} / \mathrm{s} / \mathrm{Hz} / \mathrm{cell}$-site, where the cell-cite is the area of a macrocell site.

The joint capacity of two-tier wireless network is given by $C_{m}+\sum_{i=1}^{n_{f}} C_{f i}$ where $C_{m}$ is the throughput achievable by a macrocell, $C_{f}$ is the throughput achievable by $i$ th femtocell, and $n_{f}$ is the total number of femtocells in a macrocell. Then the ASE of two-tier wireless network can be represented as

$$
\eta=\frac{C_{m}+\sum_{i=1}^{n_{f}} C_{f i}}{\pi R^{2} w_{t}}
$$

where $R$ is the radius of macrocell, and $w_{t}$ denotes the total amount of available spectrum for the two-tier wireless network.

Furthermore, the achievable throughput of macrocell (femtocell) can be estimated by the Shannon formula

$$
C=w \log _{2}(1+\gamma)
$$

where $C$ is achievable throughput, $w$ is the amount of spectrum available, and $\gamma$ is the received signal-tointerference-plus-noise ratio (SINR). Note that (2) regards the interference as white Gaussian noise and gives the worst-case estimate of achievable capacity.

Assume that the total amount of licensed spectrum is $w_{t} \mathrm{~Hz}$. With co-channel spectrum usage, each tier can use all $w_{t} \mathrm{~Hz}$, i.e., $w=w_{t}$; With dedicated-channel spectrum usage, $w_{t} \mathrm{~Hz}$ spectrum is partitioned to two spectrum potions of $w_{m} \mathrm{~Hz}$ and $w_{f} \mathrm{~Hz}$ (i.e., $w_{m}+w_{f}=w_{t}$ ), where $w_{m}$ and $w_{f}$ are the dedicated amounts of spectrum for macrocell and femtocell, respectively.

Comparing two traditional strategies of spectrum arrangement, there are pros and cons of them from the perspective of achievable network capacity. With co-channel spectrum usage, each radio tier is granted largest amount of available spectrum, $w_{t}$, but the cochannel frequency reuse in two tiers imposes severe cross-tier interferences and consequently is considered the highest risk deployment. In this case, the SINR value of $\gamma$ is lower than that with dedicated-channel spectrum usage, and it can be deduced from (2) that higher crosstier interference tends to become the capacity-limiting factor in this case. On the other hand, the cross-tier interference is lower with dedicated-channel spectrum usage, and the SINR is higher than that with co-channel spectrum usage. Whereas the amount of available spectrum is reduced at each tier as $w_{m}<w_{t}$ and $w_{f}<w_{t}$. It can be deduced from (2) that the reduced amount of spectrum tends to become the capacity-limiting factor in this case.

\subsection{Spectrum usage selection to improve spectrum utilization}

As we discussed, the cross-tier interference impacts the overall network capacity. On generating the cross-tier interference, macrocell and femtocell can play the role of either the interfering party (aggressor) or the interfered party (victim). As listed in Table 1, the cross-tier interferences can be classified accordingly to four interference scenarios: the interference scenario I-the uplink interference from HUE (aggressor) to eNB (victim), the interference scenario II-the downlink interference from 
Table 1 Cross-tier interference scenarios in two-tier LTE network

\begin{tabular}{lcc}
\hline Number & Aggressor & Victim \\
\hline I & HUE & eNB uplink \\
II & HeNB & eNB downlink \\
III & MUE & HeNB uplink \\
IV & eNB & HeNB downlink \\
\hline
\end{tabular}

HeNB (aggressor) to MUE (victim), the interference scenario III-the uplink interference from MUE (aggressor) to HeNB (victim), and the interference scenario IV-the downlink interference from eNB (aggressor) to HUE (victim) [2]. Moreover, the cross-tier interference experienced by a specific femtocell is different from one another. It is apparent that the severeness of cross-tier interference is relevant to the spatial condition of femtocell-the relative location between a HeNB and an eNB. For instance, the relative location affects the interference scenarios IV and III. The closer a HeNB locates to its embedding eNB, the higher the cross-tier interference in the interference scenario IV and III.

Since the femtocells located at different locations experience different cross-tier interference, the achieved capacities of them are different as well. For the femtocells close to the embedding eNB, the cross-tier interference tends to be the capacity-limiting factor; whereas for the femtocells at the cell edge of a macrocell, the spectrum available tends to be the capacity-limiting factor. To increase overall spectrum utilization, it would be better off if each HeNB can select its operation mode from two traditional spectrum usage modes to avoid whichever of the available bandwidth and the cross-tier interference becoming the capacity-limiting factor. In other words, a HeNB should be able to operate in either the mode of co-channel spectrum usage or the mode of dedicatedchannel spectrum usage. The selection should be based on a proper criterion, which is presented in the following section.

\subsection{Criterion of selecting spectrum usage mode by femtocells}

To improve overall spectrum utilization, the major objective of usage mode selection is to maximize the ASE as represented in (1), which is equivalent to maximize joint two-tier capacity $C_{m}+\sum_{i=1}^{n_{f}} C_{f i}$. In addition to maximizing the ASE, another aspect to determine the spectrum arrangement is the achievable throughput at each tier. The joint two-tier network capacity can be higher with a selected usage mode, whereas the achievable throughput of one tier $\left(C_{m}\right.$ or $\left.C_{f}\right)$ may be too low to support enough services for mobile users. Therefore, each tier should provide a minimum prescribed throughput.
From the above discussion, the criterion of spectrum arrangement for two-tier LTE cellular network needs to take into account both the joint two-tier capacity and the required capacity at each radio tier. Hence, instead of adopting the criterion to solely maximize the joint two-tier capacity, we adopt a criterion of selecting the spectrum usage mode as follows: The co-channel spectrum usage is chosen only when both macrocell and femtocell prefer it in terms of higher capacity; otherwise, dedicatedchannel spectrum usage is chosen. This criterion is a suboptimal with respect to the joint capacity of macrocell and femtocell in all circumstances, but it is practical one as it excludes the involvement of collecting relevant information for calculating joint two-tier capacity. Furthermore, higher achieved capacities at both macrocell tier and femtocell tier imply that the cross-tier interference is not the dominating factor that limits the network capacity.

\section{Hybrid spectrum arrangement for two-tier LTE cellular network}

\subsection{Hybrid spectrum arrangement and its implementation}

The two traditional spectrum usages result in disparate amounts of spectrum and cross-tier interferences. For selecting a proper spectrum usage mode, a femtocell needs to trade off the amount of available spectrum and the experienced cross-tier interference caused by two spectrum usage modes. Assuming the value of $w_{t}$ with co-channel spectrum usage and the values of $w_{m}$ and $w_{f}$ with dedicated-channel spectrum usage are known, we now examine the effects of cross-tier interference on the femtocell's decision making. Since the cross-tier interference is largely dependent on the spatial condition of a femtocell, the criterion of spectrum usage selection can be translated to its relevant spatial condition-the location of HeNB to eNB. According to their spatial conditions, the femtocells are distinguished to be inner and outer femtocells. The HeNBs of inner and outer femtocells operate in dedicated-channel spectrum usage and in co-channel spectrum usage, respectively. With above discussion, hybrid spectrum arrangement is a natural consequence when multiple femtocells within a macrocell select their spectrum usage mode by self-configuration.

Denote the distance between a HeNB and its embedding eNB as $d$, and denote the distance threshold to differentiate inner and outer femtocells as $d_{\mathrm{th}}$. As shown in Figure 1, a femtocell is viewed as an inner femtocell when a HeNB is located at the position where $d \leq d_{\mathrm{th}}$; otherwise, it is viewed as an outer femtocell.

Since inner and outer femtocells adopt different spectrum usage modes, the spectrum usage modes of femtocells within a macrocell become mixed. Hence, the proposed method for spectrum arrangement is called hybrid spectrum arrangement in this article. As shown 


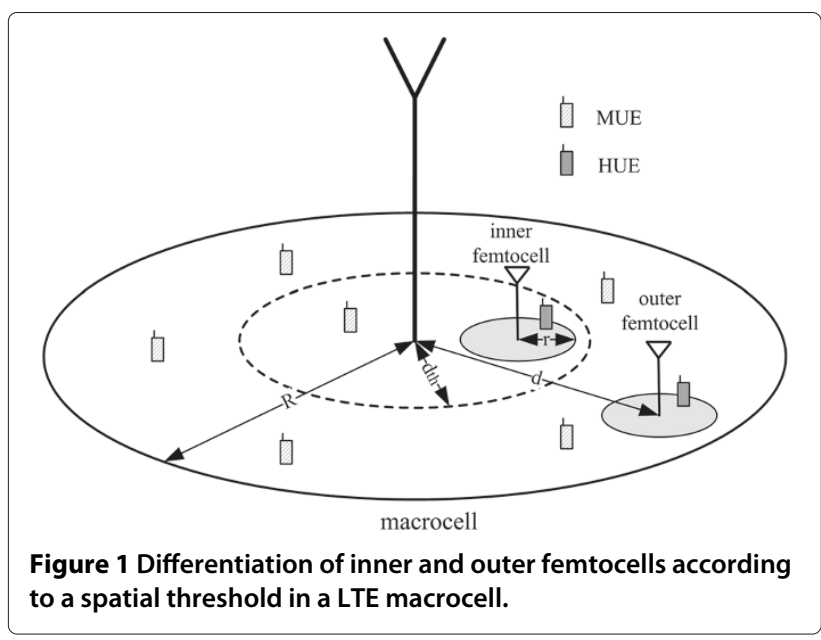

in Figure 2, with hybrid spectrum arrangement, $w_{t} \mathrm{~Hz}$ spectrum is partitioned to two spectrum potions, $w_{m} \mathrm{~Hz}$ and $w_{f} \mathrm{~Hz} ; w_{m}$ is assigned to macrocell, $w_{f}$ is assigned to inner femtocells, and $w_{t}$ can be used by outer femtocells.

In practical implementation, the parameter of $d$ can be estimated at $\mathrm{HeNB}$ corresponding to the received pilot power of eNB, i.e., reference signal received power (RSRP) in LTE network. The RSRP can be estimated by the added functionality at HeNB or can be estimated by HUE and sent to HeNB as a measurement report via an added radio resource control (RRC) signalling message. In this way $d_{\text {th }}$ corresponds to a pilot power threshold, $p_{\text {th }}$. Denote the estimated value of RSRP as $p_{r}$. A femtocell is viewed as an inner femtocell if $p_{r} \geq p_{\text {th }}$; otherwise, it is viewed as an outer femtocell. Using $p_{\text {th }}$ for decision making is regarded as a better approach rather than using $d_{\text {th }}$ because $p_{r}$ reflects the actual link quality with pathloss and shadowing between a HeNB and its embedding eNB. Assume the spectrum usage mode is allowed to be determined separately on LTE downlink and uplink, the values of $d_{\text {th }}$ for downlink and uplink can be set to different values.

The procedure to determine the spectrum usage mode by a HeNB is shown in Figure 3. Firstly, a HeNB detects

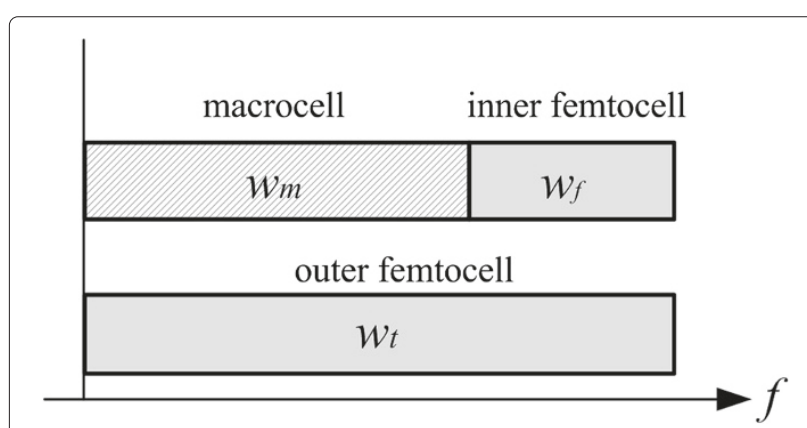

Figure 2 Hybrid spectrum arrangement for LTE macrocell and femtocells.

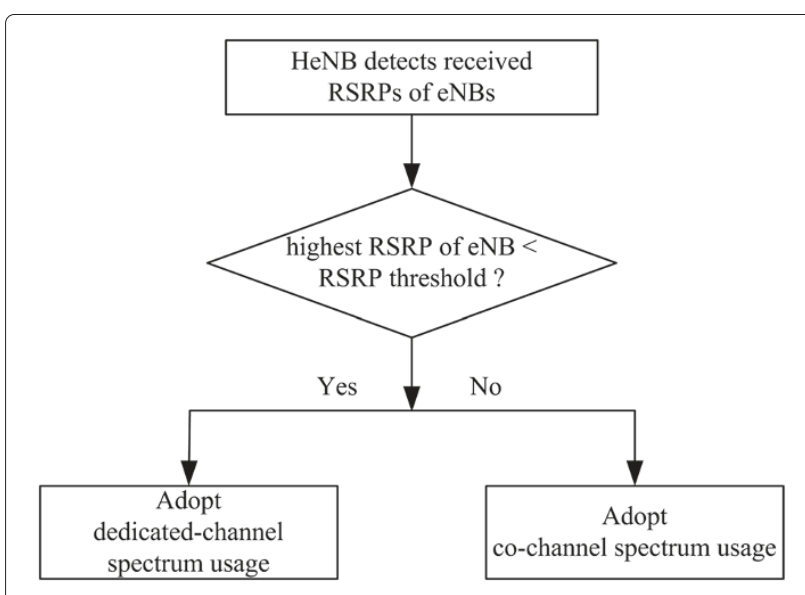

Figure 3 Procedure to determine the spectrum usage mode by HeNB.

the received RSRPs of neighboring eNBs. The highest detected value of RSRP of an eNB $\left(p_{r}^{\max }\right)$ is thought to be received from the embedding eNB with respect to the HeNB. Then, the HeNB compares $p_{r}^{\max }$ with $p_{\text {th }}$, and determines the adopted spectrum usage mode based on the comparison result.

\subsection{Downlink and uplink analysis to determine the spatial thresholds}

Next, we clarify how to determine the values of spatial threshold $d_{\text {th }}$ on downlink and uplink for selecting the spectrum usage mode. In the analysis, we consider the case of $n_{f}=1$, i.e., only one femtocell exists within a macrocell. For the case of $n_{f}>1$, i.e., multiple femtocells exist within a macrocell, we evaluate the performance by system-level simulation.

\subsubsection{Downlink analysis}

First, we evaluate the downlink throughput of macrocell. According to Shannon formula, the downlink throughput achieved by macrocell depends on the bandwidth and SINR. The downlink throughput of macrocell with co-channel spectrum usage, $C_{m}^{d l, c o-c h a n n e l}$, and that with dedicated-channel spectrum usage, $C_{m}^{d l, \text { dedicated, }}$ can be estimated by

$$
C_{m}^{d l, \text { co-channel }}=w_{t} \log _{2}\left(1+\gamma_{m}^{d l, \text { co-channel }}\right)
$$

and

$$
C_{m}^{d l, \text { dedicated }}=w_{m} \log _{2}\left(1+\gamma_{m}^{d l, \text { dedicated }}\right)
$$

where $\gamma_{m}^{d l, c o-c h a n n e l}$ and $\gamma_{m}^{d l, \text { dedicated }}$ denote the downlink SINRs of MUEs with co-channel spectrum usage and dedicated-channel spectrum usage, respectively. Note that the downlink throughput of macrocell should be estimated as the sum of achievable throughput of assigned 
resource blocks (RBs) of MUEs. The sum computation is omitted here and hereafter for terminology simplicity.

The downlink SINR of MUE, $\gamma_{m}^{d l}$, can be evaluated by

$$
\begin{aligned}
\gamma_{m}^{d l} & =\frac{s_{m}}{I_{m m}^{d l}+I_{f m}^{d l}+N} \\
& =\frac{p_{t, m} x_{0}^{-\alpha_{1}}}{\sum_{i=1}^{n_{m}-1} p_{t, m} x_{i}^{-\alpha}+p_{t, f} l^{-\alpha_{2}} 10^{-\frac{\beta}{10}} 10^{-\frac{\rho}{10}}+w N_{0}}
\end{aligned}
$$

where

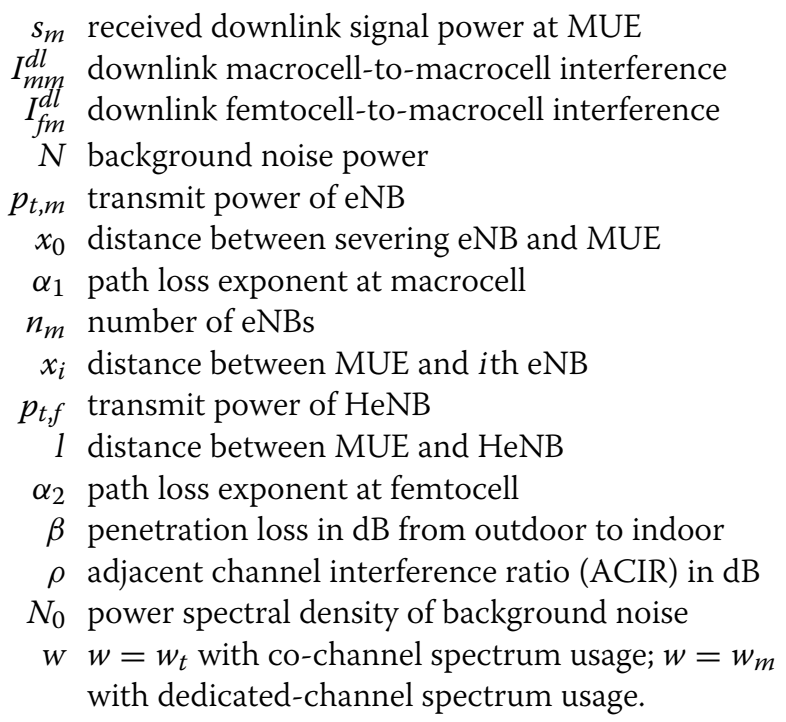

Here the downlink interference imposed on MUEs includes both intra-tier interference from neighboring macrocells, $I_{m m}^{d l}$, and cross-tier interference from femtocells, $I_{f m}^{d l}$. The cross-tier interference with the dedicatedchannel spectrum usage is assumed to be adjacent channel interference, which is reduced by adjacent channel interference ratio (ACIR, typically over $40 \mathrm{~dB}$ ), compared to the co-channel interference with co-channel spectrum usage.

Since macrocell downlink is an interference-limited system, we have $I_{m}^{d l} \gg N$. Due to the fact that the transmit power of HeNB is much less than that of eNB $\left(p_{t, f} \ll\right.$ $\left.p_{t, m}\right)$ and additional wall penetration loss $\beta$ (typically $10 \sim 20 \mathrm{~dB})$ on $p_{t, f}, I_{f m}^{d l} \ll I_{m m}^{d l}$. Thus, $\gamma_{m} \approx \frac{s_{m}^{d}}{I_{m}^{d}}$, and $\gamma_{m}^{d l \text {,co-channel }} \approx \gamma_{m}^{d l \text {,dedicated }}$. With $w_{t}>w_{m}$, we have $C_{m}^{d l \text {,co-channel }}>C_{m}^{d l \text {,dedicated }}$ by comparing (3) and (4). The interpretation of the analysis result is as follows. Due to the fact that the transmit power of HeNB is much less than that of eNB and the penetration loss of walls in femtocell, the co-tier interference from other macrocells is the dominant downlink interference for outdoor MUEs rather than the cross-tier interference from femtocells. Thus, larger bandwidth with the co-channel spectrum usage helps to increase the capacity of macrocells rather than the dedicated-channel spectrum usage. Hence, macrocell prefers the co-channel spectrum usage on downlink to maximize its capacity for the MUEs on average. Therefore, to satisfy the our proposed criterion for usage selection, the decision of selection on downlink depends on comparing the achieved throughputs of femtocell under two spectrum usage modes.

Next, we evaluate the downlink throughput of femtocell. The downlink throughput of femtocell with co-channel spectrum usage, $C_{f}^{d l \text {,co-channel }}$, and that with dedicatedchannel spectrum usage, $C_{f}^{d l \text {,dedicated }}$, can be estimated by

$$
C_{f}^{d l, \text { co-channel }}=w_{t} \log _{2}\left(1+\gamma_{f}^{d l, \text { co-channel }}\right)
$$

and

$$
C_{f}^{d l, \text { dedicated }}=w_{f} \log _{2}\left(1+\gamma_{f}^{d l, \text { dedicated }}\right)
$$

where $\gamma_{f}^{d l \text {,co-channel }}$ and $\gamma_{f}^{d l \text {,dedicated }}$ denote the downlink SINRs of HUEs with co-channel spectrum usage and dedicated-channel spectrum usage, respectively.

The downlink SINR of HUE, $\gamma_{f}^{d l}$, can be evaluated by

$$
\begin{aligned}
\gamma_{f} & =\frac{s_{f}}{I_{m f}^{d l}+N} \\
& =\frac{p_{t, f} r^{-\alpha_{2}}}{\left(p_{t, m} d^{-\alpha_{1}}+\sum_{i=1}^{n_{m}-1} p_{t, m} y_{i}^{-\alpha_{1}}\right) 10^{-\frac{\beta}{10}} 10^{-\frac{\rho}{10}}+w N_{0}}
\end{aligned}
$$

where

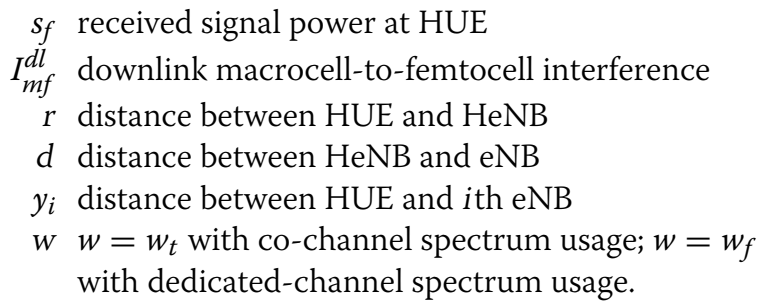

Here only cross-tier interference imposed on HUEs $\left(I_{m f}^{d l}\right)$ is considered since no co-tier interference between femtocells exists when $n_{f}=1$.

It can be observed from (8) that $\gamma_{f}^{d l}$ depends on $d$, which is the distance between a HeNB and its embedding eNB. The closer a femtocell is located from eNB, the higher the downlink macrocell interference the HUEs suffer. When a femtocell is located very close to eNB, the downlink interference dominates rather than noise, i.e., $I_{m f}^{d l} \gg N$. Due to large ACIR, $I_{m f}^{d l}$ with dedicatedchannel spectrum usage is much less than that with co-channel spectrum usage. Hence, the downlink throughput of a femtocell with dedicated-channel spectrum usage is larger than that with co-channel spectrum usage (i.e., $C_{f}^{d l, \text { dedicated }}>C_{f}^{d l, \text { co-channel }}$ ) when HeNB is 
located very close to eNB. Thus, the femtocell prefers dedicated-channel spectrum usage at a close distance to eNB. With the increase of $d$, the interference from macrocell $\left(I_{m f}^{d l}\right)$ reduces with co-channel spectrum usage due to increased pathloss, and hence the achieved capacity of femtocell $\left(C_{f}^{d l, c o-c h a n n e l}\right)$ increases. When the femtocell is located at a certain distance away from eNB such that the interference from macrocell $\left(I_{m f}^{d l}\right)$ reduces significantly, bandwidth $w$ becomes the dominant factor on determining the achievable throughput instead of $\operatorname{SINR}(\gamma)$. Consequently, $C_{f}^{d l, \text { co-channel }}>C_{f}^{d l \text {,dedicated }}$ when a HeNB locates at a certain distance away from eNB. Thus, a femtocell prefers co-channel spectrum usage when it locates at a certain distance away from eNB. The distance threshold, $d_{\mathrm{th}}^{d l}$, can be determined under the condition that the achieved downlink throughput of femtocell with co-channel spectrum usage is the same as that with dedicated-channel spectrum usage, i.e., $d=d_{\mathrm{th}}^{d l}$ if $C_{f}^{d l, \text { co-channel }}(d)=C_{f}^{d l, \text { dedicated }}(d)$.

For the case of $n_{f}>1$, our simulation result shows that the macrocell stills prefers co-channel spectrum usage with reasonable density of femtocells deployed in the macrocell. On the other hand, the femtocells can still keep the decision as $n_{f}=1$ and resort to interference mitigation method to alleviate the residual interferences.

\subsubsection{Uplink analysis}

On uplink, we first evaluate the achievable uplink throughput of femtocell. The uplink SINR of HeNB, $\gamma_{f}^{u l}$, can be evaluated by

$$
\gamma_{f}^{u l}=\frac{s_{f}^{u l}}{I_{m f}^{u l}+N}=\frac{\hat{p}_{t, f} r^{-\alpha_{2}}}{\sum_{i=1}^{n_{m}} \hat{p}_{t, m} u_{i}^{-\alpha_{1}} 10^{-\frac{\beta}{10}} 10^{-\frac{\rho}{10}}+w N_{0}}
$$

$s_{f}^{u l}$ received signal power at $\mathrm{HeNB}$

$I_{m f}^{u l}$ uplink macrocell-to-femtocell interference

$\hat{p}_{t, f}$ transmit power of HUE

$\hat{p}_{t, m}$ transmit power of MUE

$n_{m}$ number of MUEs in macrocells

$u_{i}$ distance between HeNB and ith MUE.

If no active MUE is near the HeNB, the femtocell uplink is largely a noise-limited system. Hence, $I_{m f}^{u l} \approx 0$, and $\gamma_{f} \approx \frac{s_{f}^{u l}}{N}$. Considering the throughput of femtocell on average, $C_{f}^{u l \text {,co-channel }}>C_{f}^{u l \text {,dedicated }}$. Thus, the femtocell prefers co-channel spectrum usage to maximize its capacity on uplink. Therefore, to satisfy the criterion for usage selection, the spectrum usage mode for uplink depends on the choice of macrocell.
Next, we evaluate the achievable uplink throughput of macrocell. The uplink SINR of macrocell, $\gamma_{m}^{u l}$, can be evaluated by

$$
\begin{aligned}
\gamma_{m}^{u l} & =\frac{s_{m}^{u l}}{I_{m m}^{u l}+I_{f m}^{u l}+N} \\
& =\frac{\hat{p}_{t, m} \hat{x}_{i}^{-\alpha_{1}}}{\sum_{i=1}^{n_{m o}-1} \hat{p}_{t, m} v_{i}^{-\alpha_{1}}+\sum_{i=1}^{n_{h}-1} \hat{p}_{t, f} d_{i}^{-\alpha_{1}} 10^{-\frac{\beta}{10}} 10^{-\frac{\rho}{10}}+w N_{0}}
\end{aligned}
$$

where

$n_{m o}$ number of MUEs outside of the interested eNB

$v_{i}$ distance between $i$ th MUE and the interested eNB

$n_{h}$ number of HUEs in femtocell

$d_{i}$ distance between $i$ th HUE and the interested eNB.

As $\gamma_{m}^{u l}$ is a function of $d$, the closer the femtocell is located to the eNB, the higher uplink interference the macrocell suffers. The achievable uplink throughput of macrocell with co-channel spectrum usage is less than that with dedicated-channel spectrum usage (i.e., $C_{m}^{u l, c o-c h a n n e l}<$

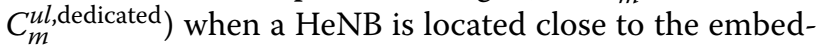
ding eNB. However, the uplink throughput in co-channel spectrum usage becomes larger than that in dedicated spectrum usage (i.e., $C_{m}^{u l \text {,co-channel }}>C_{m}^{u l \text {,dedicated }}$ ) when the $\mathrm{HeNB}$ is located at certain distance away from the embedding eNB. The specific distance that the uplink throughputs are equal with two spectrum usage modes can be set to be the uplink distance threshold, $d_{\mathrm{th}}^{u l}$, i.e., $d=d_{\text {th }}^{u l}$ if $C_{m}^{u l \text {,co-channel }}(d)=C_{m}^{u l \text {,dedicated }}(d)$ to determine the uplink spectrum usage mode.

When the HeNB is located farther than $d_{\mathrm{th}}^{u l}$, the femtocell is viewed as an outer femtocell and operates in co-channel spectrum usage; otherwise, it operates in dedicated-channel spectrum usage.

For the case of $n_{f}>1$, the aggregate interference on the macrocell uplink from both MUEs in other macrocells and HUEs needs to be considered. The interference level can be measured by interference over thermal (IoT), which is defined as the ratio of the interference plus thermal noise to thermal noise. The uplink aggregate IoT of macrocell, IoT $_{m}^{u l}$, is evaluated by

$$
\operatorname{IoT}_{m}^{u l}=10 \log _{10}\left(\frac{I_{m m}^{u l}+I_{f m}^{u l}+N}{N}\right) \mathrm{dB} .
$$

The maximal allowed aggregate interference in macrocell uplink can be set as the total IoT threshold, $\mathrm{IoT}_{\text {tot }}^{\text {th }}$. If ${ }_{\text {Io }} T_{\text {tot }}^{\text {th }}$ is per-defined, the allowed IoT introduced by femtocells, $\mathrm{IoT}_{f}^{\text {th }}$, can be calculated after taking out the current aggregate IoT at macrocell, Io $_{m}^{\text {cur }}$, from Io $_{\text {tot }}^{\text {th }}$. That is

$$
\operatorname{IoT}_{f}^{\text {th }}=10 \log _{10}\left(10 \frac{\mathrm{IoT}_{\text {tot }}^{\text {th }}}{10}-10 \frac{\mathrm{IoT}_{m}^{\mathrm{cur}}}{10}+1\right) \mathrm{dB}
$$


$\mathrm{IoT}_{f}^{\text {th }}$ can be used for deciding whether additional femtocell (when $n_{f}>1$ ) can be allowed to operate in the co-channel spectrum usage.

In LTE network, a UE may employ uplink fractional power control depending on its pathloss to the connected BS such that it does not always transmit with its full power. Hence, $\hat{p}_{t, f}$ varies according to a HUE's distance to HeNB, and $\hat{p}_{t, m}$ varies according to a MUE's distance to eNB. In the next section, we discuss that the uplink cross-tier interference from HUEs of femtocells to the eNBs (i.e., the interference scenario I) can be alleviated with proper system parameter tuning under fractional power control.

In the above analysis and the following simulation to determine the spatial thresholds, we assume the macrocells are fully loaded, i.e., all their RBs are allocated to support the traffics of MUEs. This assumption leads to a conservative decision on the value of spatial threshold, which hence gives the priority to guarantee the service provisioning of macrocells. When considering the temporal varying nature of the resource usage at macrocells, the thresholds for the spectrum usage mode selection can be made to be time variant accordingly. Hence, the femtocells can select its spectrum usage mode with respect to both temporal and spatial conditions. To implement such an approach in LTE networks, the HeNB requires more advanced hardware to detect timely resource usage of macrocell or needs message exchange between eNB and HeNB. Due to the highly dynamic nature of the resource usage of macrocell, it is hard for the HeNB to obtain timely and precise information of resource usage at macrocell. Frequent changing operation mode of femtocells also interrupts the ongoing services provided to the HUEs. A compromised approach might be more feasible: the selection of usage mode by femtocells is updated based on longer time observation of the resource usage at macrocell so that it does not change very frequently; for instance, the change of selection mode occurs only at some time between daytime and nighttime.

\section{Interference mitigation with hybrid spectrum arrangement}

With our proposed hybrid spectrum arrangement, the four interference scenarios have their distinct characteristics when comparing with pure co-channel spectrum usage and pure dedicated-channel spectrum usage. Next, we examine the severeness of four interference scenarios, identify the residual significant interference scenarios, and propose the interference mitigation methods for them.

\subsection{Discussion on interference scenario IV}

First, we give a discussion on the interference scenario IV-the downlink interference from eNB to HUEs. With our proposed hybrid spectrum arrangement, a HeNB operates in the dedicated-channel spectrum usage when it is located less than $d_{\mathrm{th}}^{d l}$; otherwise, it operates in the co-channel spectrum usage. Hence, the HUEs in the inner femtocells do not experience much downlink interference from eNB. Though the HUEs in the outer femtocells suffer downlink interference from eNBs, the gain of larger bandwidth in outer femtocells surpasses the drawback of interference such that outer femtocells can still achieve higher downlink capacity with co-channel spectrum usage. Thus, the interference scenario IV has been mitigated with hybrid spectrum arrangement.

\subsection{Discussion on interference scenario III}

With hybrid spectrum arrangement, an eNB operates in dedicated-channel spectrum usage with $w_{m} \mathrm{~Hz}$ when an inner femtocell exists. The spectrum used by the outer femtocells $\left(w_{t}\right)$ can be distinguished to two portions, MUE-absent portion $\left(w_{f}\right)$ and MUE-possible portion $\left(w_{m}\right)$. The MUE-absent portion can only be assigned to HUEs by HeNB; it can not be assigned to MUEs by eNB. On the other hand, the MUE-possible portion used by outer femtocells is overlapped with macrocell and the resource in this portion can be assigned to MUEs by eNB. Therefore, a HeNB always have MUE-absent portion $\left(w_{f}\right)$ as "clean" resource to be assigned to its HUEs. When an MUE associated with an eNB is near a CSG HeNB, the $\mathrm{HeNB}$ can perform RRM to reallocate the resource used by HUEs to MUE-absent portion. In this way the interference scenario III (the uplink interference from MUE to $\mathrm{HeNB}$ ) can be easily mitigated with hybrid spectrum arrangement.

\subsection{Interference mitigation method for interference scenario II}

The interference scenario II refers to the interference from downlink HeNB to MUE. With hybrid spectrum arrangement, different portions of spectrum are dedicated to macrocells and inner femtocells. Thus, an MUE does not suffer much downlink interference from a HeNB when it lies within the coverage an inner femtocell, regardless of whether the femtocell operates in the closed-access or open-access manner. Whereas when a MUE lies within the coverage of a closed-access outer femtocell and its ID is not in the authorized list of the CSG femtocell, the MUE suffers high downlink interference from HeNB because the spectrum used by the outer femtocell is overlapped with that used by MUEs. The problem is severer when an MUE lies within the indoor coverage of outer femtocell due to the near-far effects. In this case, an indoor MUE at the macro cell edge receives highly attenuated signal from the eNB with wall penetration loss, but receives excessive interference signal originating from a HeNB directly without such penetration loss. Consequently, "dead zone" exists for an MUE when it lies too close to an indoor HeNB: a connection can not be established with eNB or 
an ongoing connection between an MUE and its serving eNB can be interrupted.

To give priority of QoS assurance for MUEs, the severe interference scenario II should be nullified. To fulfil this task, both the serving eNB and the interfering HeNB can perform radio resource management (RRM) operations (e.g., smart resource scheduling) for the interfered MUE. The serving eNB can resort to channel-dependent scheduling for the interfered MUE. However, if all the RBs (Resource Blocks) in the MUE-possible portion $\left(w_{m}\right)$ are used by the serving eNB, no "clean" RBs are available at the serving eNB to be reallocated for the interfered MUE. On the other hand, the interfering HeNB is not aware of the existence of the near-by interfered MUEs and it can not perform RRM operations even if not all the available RBs are used.

In this article, it is proposed that a HeNB can be notified that it is interfering a MUE and then reallocates its resource to mitigate the downlink interference imposed on the MUE. In the following, we describe two schemes to fulfil the task of notification of near-by MUE to the interfering HeNB. The first scheme is implemented over wired networks, and the other scheme is implemented over the air.

\subsubsection{Notification of interfered MUE to HeNB over wired networks}

The first method to notify a HeNB that it interferes a near-by MUE is implemented over wired networks. The procedure is shown in Figure 4. When the downlink performance of MUE degrades by detecting the increase of packet loss rate and packet delay, the MUE checks its experienced downlink IoT. If the change of downlink IoT is larger than a threshold, the MUE attributes the observed interference to the existence of near-by HeNB after double checks: comparing the estimated RSRP of HeNB with a pre-defined threshold and confirming that HeNB's physical cell identifier (PCI) is within csg-PhysCellIdRange. If multiple HeNBs are detected by the interfered MUE, the HeNB with the highest estimated RSRP is viewed as the interfering HeNB. After detecting the interfering HeNB, the MUE sends the serving eNB a measurement report containing the HeNB's PCI information. When PCI confusion occurs for the interfering HeNB, the serving eNB asks the MUE to further read the E-UTRAN cell global identifier (ECGI) of the interfering HeNB. After that, the serving eNB sends the interfering HeNB a message, named as MUE ARRIVAL, to indicate that an MUE is near-by from it and being interfered. The message of MUE ARRIVAL is routed via mobility management entity (MME) and sent to the interfering HeNB over S1 Interface.

When a HeNB receives a message of MUE ARRIVAL, it is aware that a near-by MUE is being interfered and then it performs RRM operations to release resource for the interfered MUE. The RRM operations can be conducted in the following steps. First, it can reallocate some of the RBs used by HUEs to the MUE-absent portion; second, it can reduce the RBs that are in the MUE-possible portion used for non-real-time traffics by HUEs; third, it can reduce the RBs that are in the MUE-possible portion used for real-time traffics by HUEs if the QoS of HUEs is over qualified or can be downgraded.

When the MUE changes to IDLE mode from ACTIVE mode, or the estimated RSRP of the interfering HeNB is less than a pre-defined threshold, the MUE sends another measurement report to its serving eNB, and then the serving eNB sends the interfering HeNB a message, named as MUE DEPARTURE, to indicate that the interfered MUE is not suffering the interference from it anymore. When a HeNB receives a message of MUE DEPARTURE, it is aware that an interfered MUE moves away and it can use all the available spectrum for its HUEs.

The approach of notifying interfered MUE to HeNB over wired networks does not require additional hardware of HeNBs, but has a larger delay than the other approach, which we present next.

\subsubsection{Notification of interfered MUE to HeNB over the air}

The other scheme to notify a HeNB that it interferes a near-by MUE is implemented over the air. The procedure is shown in Figure 5. In this scheme, some subcarriers of a $\mathrm{RB}$ in the MUE-absent spectrum portion $\left(w_{f}\right)$ is reserved for notifying HeNB that an MUE is suffering its downlink interference. The HeNBs of outer femtocells do not allocate this RB to HUEs for the data transmission; the HeNBs of inner femtocells can still use the reserved RB. When a MUE is interfered by a HeNB, the MUE sends a signal, as MUE ARRIVAL, on a prescribed subcarrier of the dedicated RB. When the MUE changes to IDLE mode from ACTIVE mode, or the estimated RSRP of the interfering HeNB is less than a pre-defined threshold, the MUE sends another signal, as MUE DEPARTURE, on another prescribed subcarrier of the dedicated $R B$. To detect the notification signals, the HeNB performs energy detection on the reserved RB. Instead of using message exchange between MUE and HeNB, this scheme can be easily implemented without the requirement of strict synchronization between the MUE and the interfering HeNB.

The delay of notification over the air is the air propagation delay from an MUE to HeNB which is negligibly small, but this approach has resource overhead since it needs to reserve a RB for accomplishing the notification task.

\subsection{Interference mitigation method for interference scenario I}

The interference scenario I refers to the interference from uplink HUEs to eNB. When there are multiple HeNBs, the 


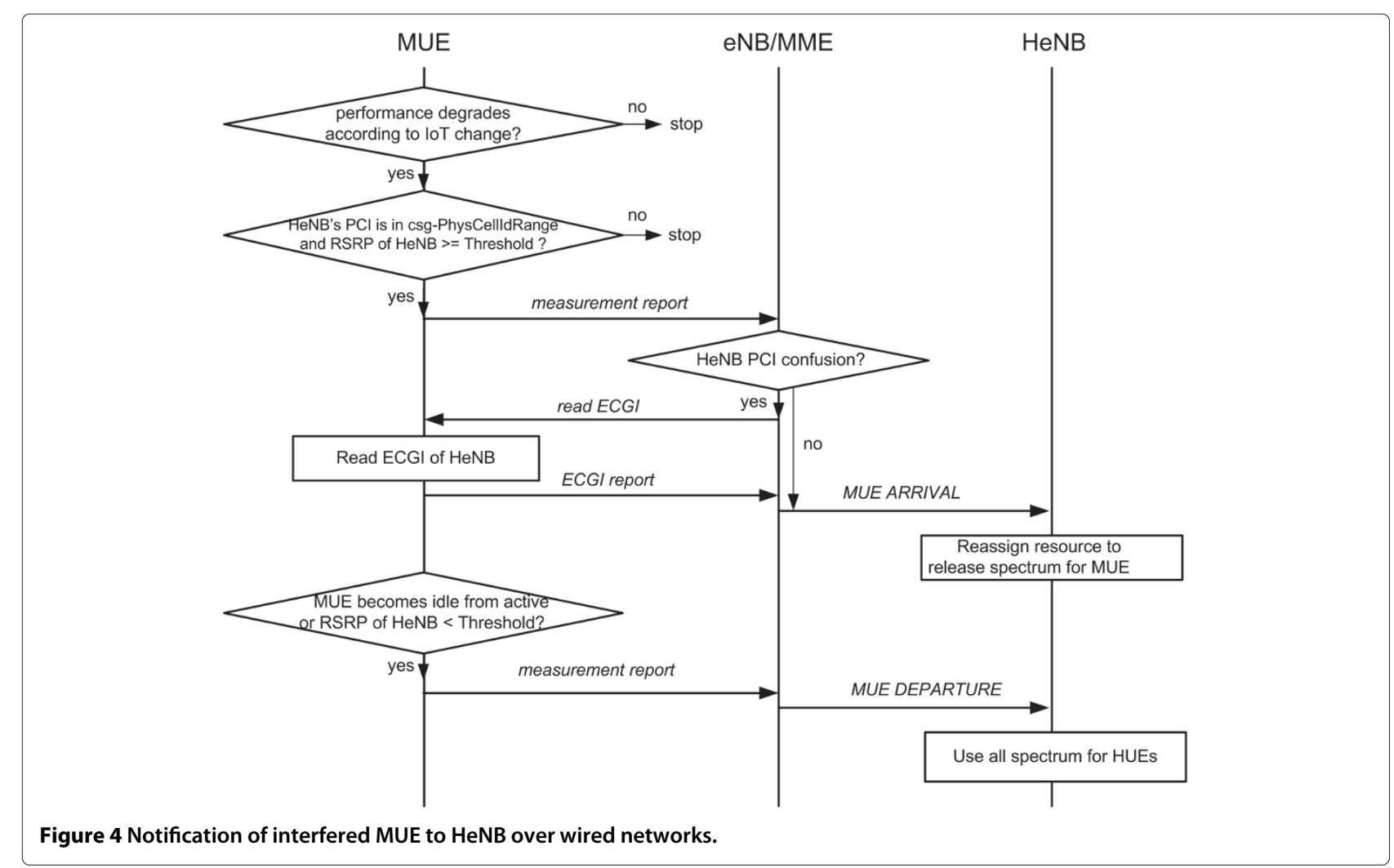

impact of the cross-tier interference from HUEs on eNB uplink performance can not be neglected with fractional uplink power control. Uplink fractional power control lets a UE transmit at a power level depending on its pathloss to BS. The formula for fractional uplink power control is $P=\min \left\{P_{\max }, P_{0}+10 \log _{10} M+\alpha P L\right\}$ where $P_{\max }$ is UE's maximum transmit power, $P_{0}$ is cell/UE specific value, $M$ is the number of assigned RBs, $P L$ is the pathloss from $\mathrm{UE}$ to $\mathrm{eNB} / \mathrm{HeNB}$, and $\alpha$ is pathloss compensation factor [27]. In our simulation evaluated in Section $6, P_{0}$ is first set to $-60 \mathrm{dBm}$, and $\alpha$ is set to 0.6 . It is seen that the uplink throughput of eNB reduces with the increase of number of HeNBs. However, if $P_{0}$ is set to $-75 \mathrm{dBm}$ for the HUEs, the imposed interference on eNB uplink is reduced significantly while the HUEs can still maintain their performance. The simulation result shows that the achieved throughput of eNB does not vary much with the increase of number of HeNBs when $P_{0}$ is set to $-75 \mathrm{dBm}$ for HUEs. Thus, different values of $P_{0}$ for macrocell and femtocell are recommended to be used in the practical system as a means to alleviate the cross-tier interference scenario I.

\section{Performance evaluation by system-level simulation}

The simulation parameters are given in Table 2, which are in line with 3GPP LTE specifications $[2,28,29]$. In our simulation, the overlaying LTE network consists of macrocells and femtocells. The simulated network layout assumes a hexagonal grid with 19 macrocell BSs (eNBs) and 3 sectors per eNB with a center-excited structure. The central macrocell is a reference cell-site when collecting performance results; other 18 macrocells act two rings of interferers to the central macrocell. The cell radius of macrocells is $500 / \sqrt{3} \simeq 289 \mathrm{~m}$, and the femtocells are assumed to have a circular coverage with radius $10 \mathrm{~m}$. The horizontal antenna pattern of macrocell is set to be $A(\varphi)=-\min \left[12\left(\frac{\varphi}{\varphi_{3} \mathrm{~dB}}\right)^{2}, A_{m}\right]$, where $\varphi_{3 \mathrm{~dB}}=70$ degrees, $A_{m}=25 \mathrm{~dB}$; the antenna pattern of femtocell is set to be omnidirectional, i.e., $A(\varphi)=0 \mathrm{~dB}$.

For the case of $n_{f}=1$, there is only one HeNB that is located in the central macrocell with distance, $d$, to the central eNB. For the case of $n_{f}>1$, there are $n_{f}$ HeNBs that are located randomly in each macrocell of all 19 macrocells. The number of active MUEs is 10 per sector in macrocell and the number of active HUEs is 4 per femtocell. The active UEs, whose number is decided in the initialization phase and kept constant for the whole simulation time, are uniformly distributed over the network area. A single transmit antenna at the UE and two receive antennas at the $\mathrm{BS}$ are used with maximal ratio combining (MRC). With co-channel spectrum usage, the available bandwidth for macrocell and femtocell are both $20 \mathrm{MHz}$. With dedicated-channel spectrum usage, the available bandwidth for macrocell and femtocell is 


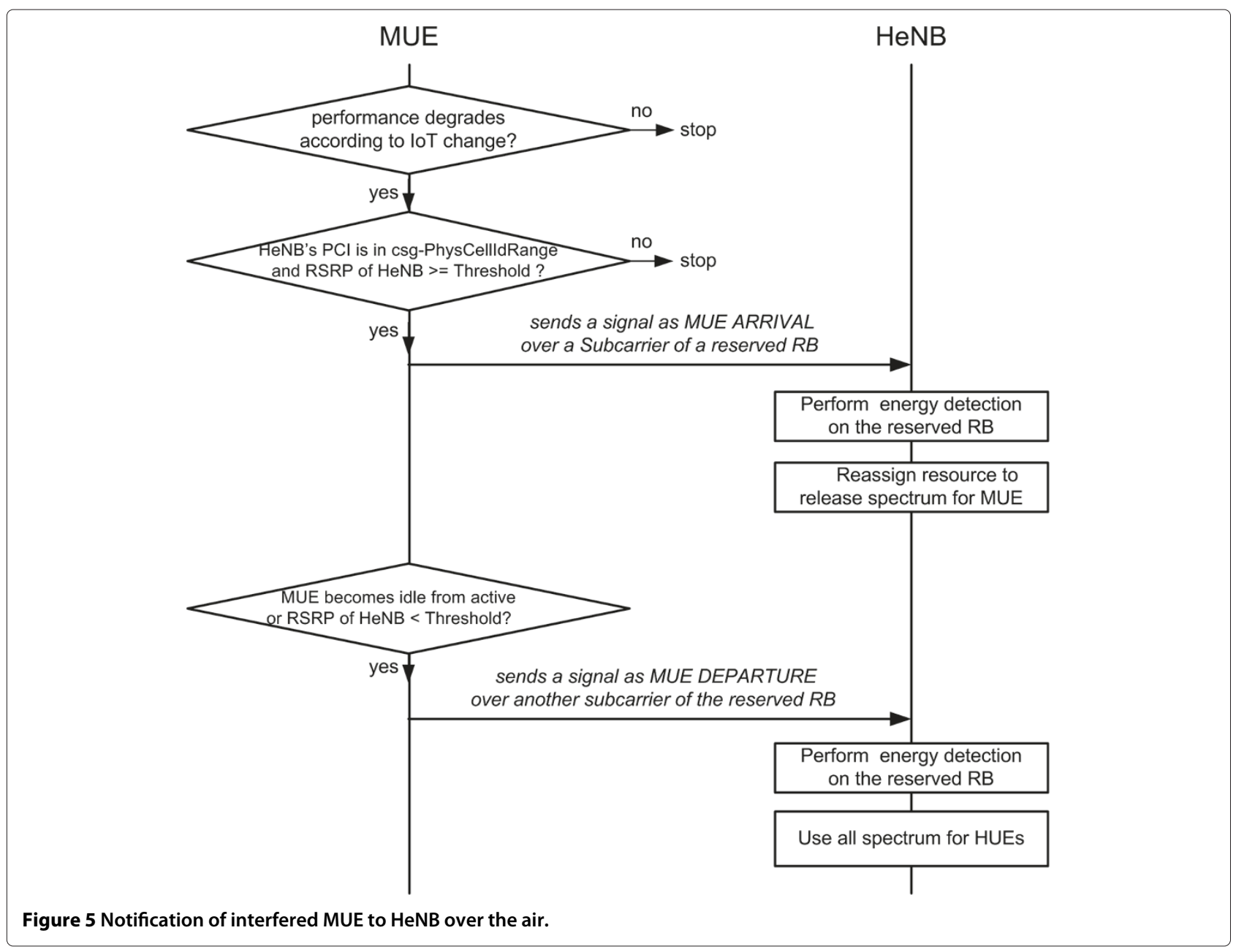

15 and $5 \mathrm{MHz}$, respectively. The cross-tier interference in the dedicated-channel spectrum usage is assumed to be adjacent-channel interference, and the ACIR is set to $45 \mathrm{~dB}$ for all the RBs in the adjacent $5 \mathrm{MHz}$. Furthermore, we assume that $10 \%$ of the total BS transmit power is allocated for RSRP signal. The overall signaling overhead is assumed to be $30 \%$. For instance, the total number of subcarriers is 1333 with $20 \mathrm{MHz}$ available bandwidth and $1.5 \mathrm{kHz}$ subcarrier spacing, and only 1200 subcarriers among them are used for data transmission. In the system simulation, when converting a calculated SINR to the throughput of macrocell or femtocell, the attenuated and truncated forms of the Shannon bound are employed on downlink and uplink as specified in [28].

First, the simulation is performed for the case of $n_{f}=1$, i.e., only one femtocell is placed within the central macrocell. Figure 6 shows the downlink per-sector throughput of central eNB versus the location of HeNB. The throughput of eNB with co-channel spectrum usage, $C_{m}^{d l, \text {,co-channel and }}$ that with dedicated-channel spectrum usage, $C_{m}^{d l \text {,dedicated, }}$ are depicted as solid line and dotted line, respectively. It
Table 2 Simulation parameters

\begin{tabular}{lcc}
\hline & Macrocell & Femtocell \\
\hline System bandwidth & $20 \mathrm{MHz}$ (co-channel) & $20 \mathrm{MHz}$ (co-channel) \\
Cell layout & $15 \mathrm{MHz}$ (dedicated) & $5 \mathrm{MHz}$ (dedicated) \\
& Hexagonal grid, 19 BSs, & Circular cell, \\
Antenna gain & $\mathrm{BS}: 15 \mathrm{dBi}, \mathrm{UE}: 0 \mathrm{dBi}$ & $\mathrm{BS}: 5 \mathrm{dBi}, \mathrm{UE}: 0 \mathrm{dBi}$ \\
Cell radius & $289 \mathrm{~m}$ & $10 \mathrm{~m}$ \\
Pathloss & $128.1+37.6 \log _{10} \mathrm{~d}$, & $127.0+30 \log _{10} \mathrm{~d}$ \\
& $\mathrm{~d}$ in [km] & $\mathrm{d} \mathrm{in}[\mathrm{km}]$ \\
Lognormal shadowing & $8 \mathrm{~dB}$ & $10 \mathrm{~dB}$ \\
Penetration loss & $20 \mathrm{~dB}$ & $20 \mathrm{~dB}$ \\
BS TX power & $49 \mathrm{dBm}$ & $20 \mathrm{dBm}$ \\
UE power class & $23 \mathrm{dBm}$ & $23 \mathrm{dBm}$ \\
BS noise figure & $5 \mathrm{~dB}$ & $5 \mathrm{~dB}$ \\
UE noise figure & $9 \mathrm{~dB}$ & $9 \mathrm{~dB}$ \\
Thermal noise level & $-174 \mathrm{dBm} / \mathrm{Hz}$ & $-174 \mathrm{dBm} / \mathrm{Hz}$ \\
Number of UEs & $10 \mathrm{per} \mathrm{sector}$ & $4 \mathrm{per} \mathrm{cell}$ \\
\hline
\end{tabular}




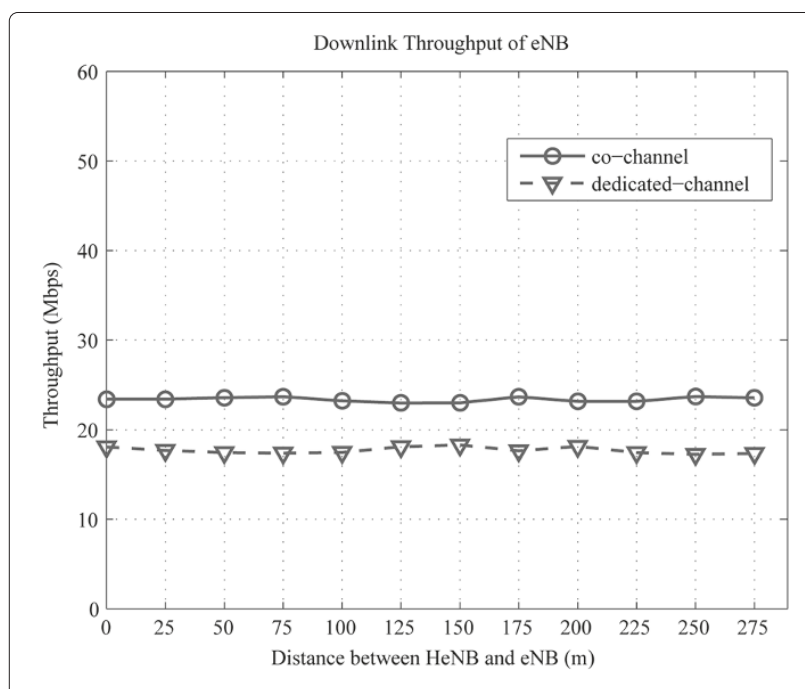

Figure 6 Downlink throughput of eNB.

is seen from Figure 6 that $C_{m}^{d l, \text { co-channel }}>C_{m}^{d l, \text { dedicated, }}$ and $C_{m}^{d l, \text { co-channel does not vary very much as the loca- }}$ tion of HeNB changes. Figure 7 shows the downlink throughputs of femtocell (denoted as $C_{f}^{d l, \text { co-channel }}$ and $C_{f}^{d l \text {,dedicated }}$ for co-channel and dedicated-channel spectrum usage modes) as we change the location of HeNB. It is seen from Figure 7 that when the HeNB is located close to eNB and less than $28 \mathrm{~m}, C_{f}^{d l, c o-c h a n n e l}<C_{f}^{d l \text {,dedicated }}$. When the HeNB is located at farther away than about $28 \mathrm{~m}$ from eNB, $C_{f}^{d l, c o-c h a n n e l}>C_{f}^{d l \text {,dedicated }}$. The distance of $28 \mathrm{~m}$ (intersection of solid line and dotted line) where $C_{f}^{d l, \text { co-channel }}=C_{f}^{d l, \text { dedicated }}$ can be set to be the downlink distance threshold, $d_{\mathrm{th}}^{d l}$, for a HeNB to determine its

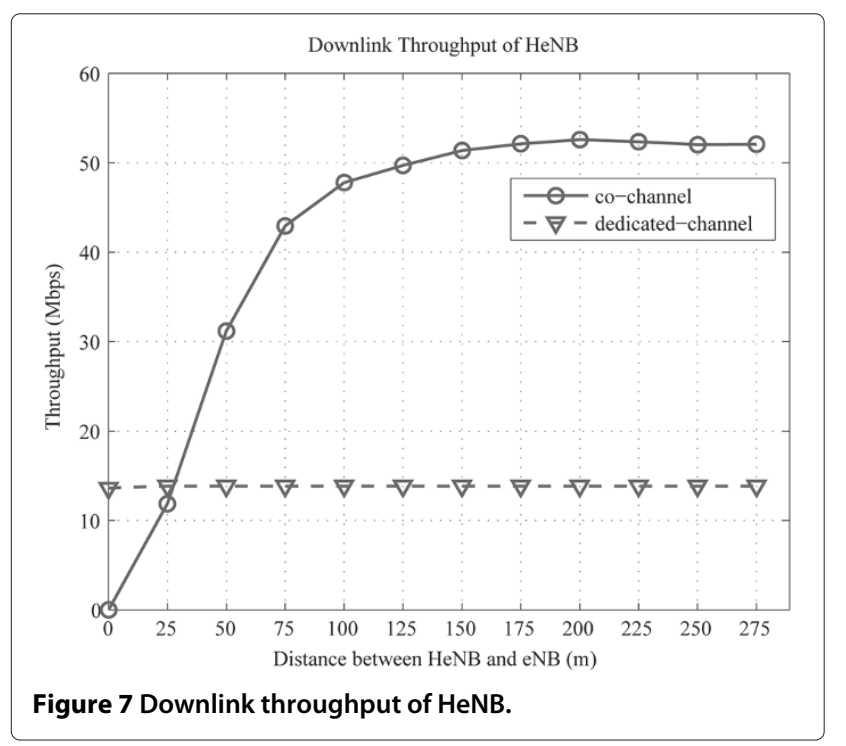

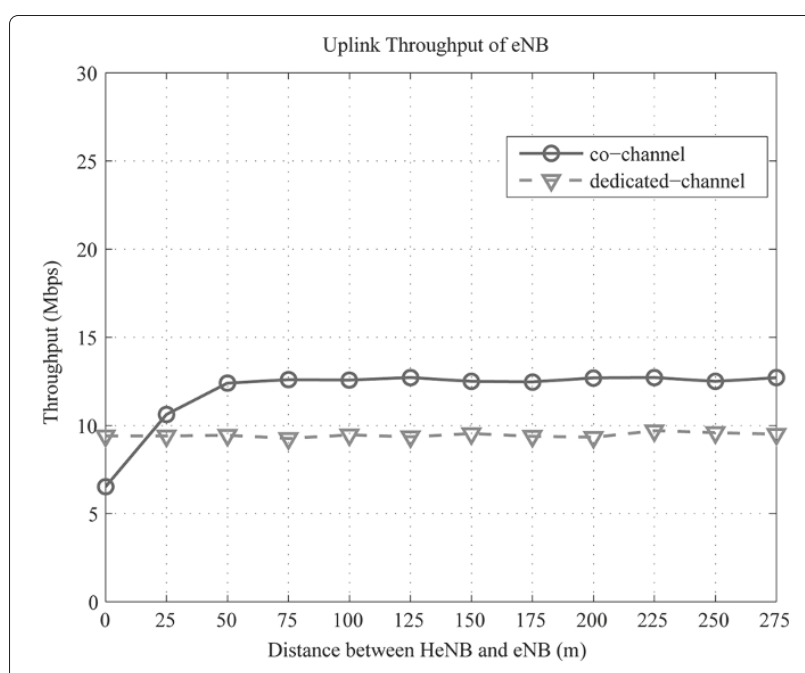

Figure 8 Uplink throughput of eNB.

spectrum usage mode. It is seen that the downlink distance threshold $\left(d_{\mathrm{th}}^{d l}\right)$ is not far from the embedding eNB such that most femtocells within a macrocell can operate in the co-channel spectrum usage mode. The corresponding downlink RSRP threshold, $p_{\mathrm{th}}^{d l}=49 \mathrm{dBm}-10 \mathrm{~dB}-$ $(128.1+37.6 \log 10(28 / 1000)) \mathrm{dB}=-30.7 \mathrm{dBm}$, where $10 \mathrm{~dB}$ is introduced as the pilot power of eNB is assumed to be $10 \%$ of the total eNB transmit power.

Figures 8 and 9 show the uplink sector throughput of macrocell and the uplink throughput of femtocell, respectively, as we change the location of HeNB. Uplink fractional power control is employed for a UE with the formula for fractional uplink power control as $P=$ $\min \left\{P_{\max }, P_{0}+10 \log _{10} M+\alpha P L\right\}$. In our simulation, $P_{0}$ is assumed to be cell specific and is set to $-60 \mathrm{dBm}$, and

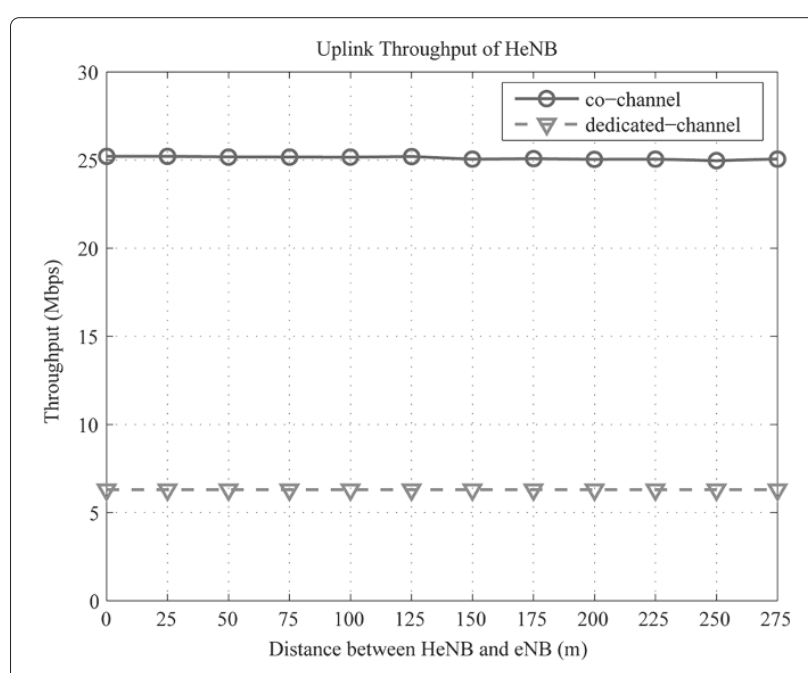

Figure 9 Uplink throughput of HeNB. 
$\alpha$ is set to 0.6. It is seen from Figure 9 that $C_{f}^{u l, \text { co-channel }}>$

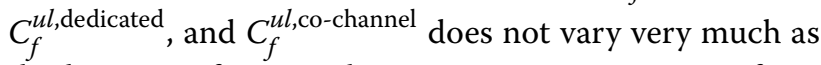
the location of HeNB changes. However, it is seen from Figure 8 that $C_{m}^{u l, \text { co-channel }}<C_{m}^{u l \text {,dedicated }}$ when the HeNB is close to eNB (less than $50 \mathrm{~m}$ away from eNB). When the HeNB is located farther away than about $50 \mathrm{~m}$ from eNB, $C_{m}^{u l \text {,co-channel }}>C_{m}^{u l \text {,dedicated }}$. The distance of $50 \mathrm{~m}$ (intersection of solid line and dotted line) where $C_{m}^{u l, \text { co-channel }}=$ $C_{m}^{u l \text {,dedicated }}$ can be set to be the uplink distance threshold, $d_{\text {th }}^{u l}$, for a HeNB to determine its spectrum usage mode.

Figure 10 shows the uplink throughput of eNB with multiple HeNBs. When there are multiple HeNBs, the impact of the cross-tier interference from HUEs on eNB uplink performance is severe if $P_{0}$ is set to $-60 \mathrm{dBm}$ for HUEs. It is seen that the uplink throughput of eNB reduces with the increase of number of HeNBs. However, if $P_{0}$ is set to $-75 \mathrm{dBm}$ for HUEs, the impact on eNB uplink is reduced significantly. It can be seen from Figure 10 that the achieved throughput of eNB does not vary much with the increase of number of HeNBs when $P_{0}$ is set to $-75 \mathrm{dBm}$ for HUEs. Thus, different values of $P_{0}$ are recommended to be used by MUEs and HUEs in the practical system as a means to mitigate the interference scenario I.

Figure 11 reveals the effects of interference scenario II between an MUE and an outer femtocell. The solid line shows the downlink throughput of an MUE without interfering HeNB with respect to the distance between the MUE and the serving eNB. The dotted lines shows the downlink throughput of the MUE with an interfering HeNB of outer femtocell with respect to different locations of the HeNB. As shown in Figure 11, the downlink throughput of MUE becomes 0 when it is at the indoor femtocell area due to high downlink interference from HeNB. Hence, the indoor femtocell area of about $10 \mathrm{~m}$ are the dead zone of MUEs. With the proposed method of

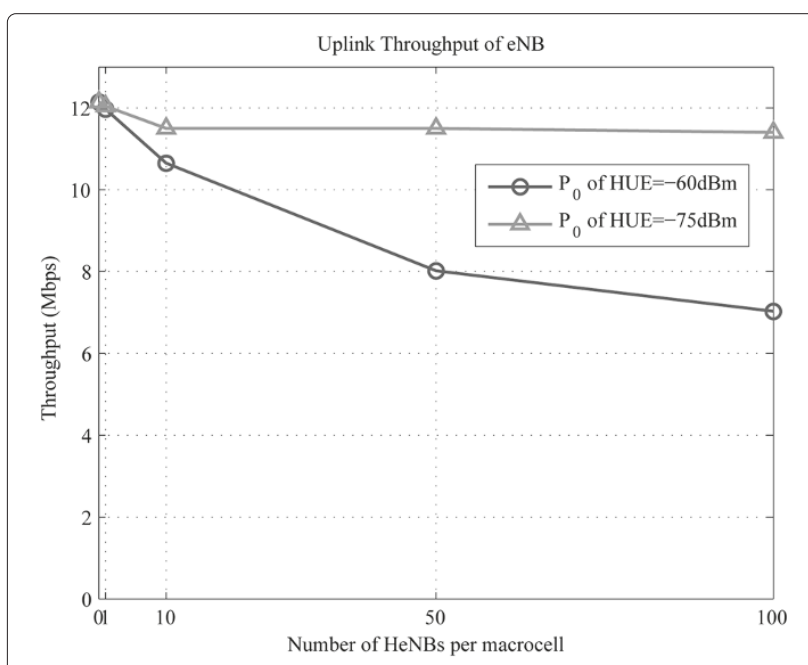

Figure 10 Uplink throughput of eNB with multiple HeNBs.

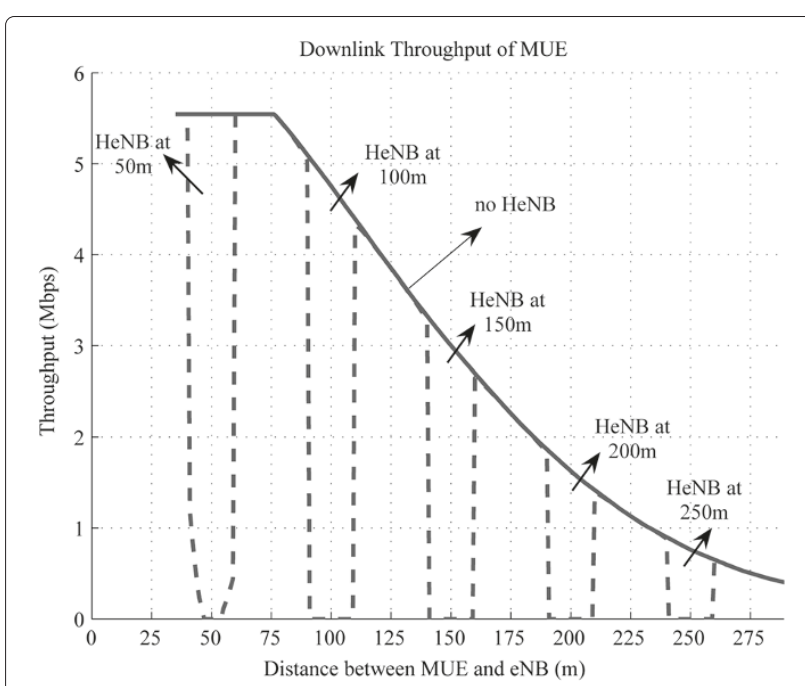

Figure 11 Downlink throughput of MUE with interfering HeNB.

notification of MUE to the interfering HeNB, the downlink throughput of MUE can be kept unchanged when it is at the indoor femtocell area since the interference can be mitigated by resource reallocation at HeNB.

\section{Conclusions}

Our study in this article started with examining the pros and cons of two traditional strategies of spectrum arrangement for two-tier LTE cellular network, and pointed out that proper spectrum arrangement needs to trade off the amount of available spectrum and cross-tier interference-either of them can be the capacity-limiting factor with the traditional strategies. The proposed hybrid spectrum arrangement in this article can properly fulfill this task. In our proposal, a femtocell selects its spectrum usage mode according to a criterion such that both macrocell and femtocell can be benefited in terms of achieved capacity. The criterion can be further translated to the spatial conditions of femtocells within macrocell. Specifically, a HeNB decides its location region by comparing the estimated RSRP of eNB at HeNB with a predefined threshold, which is determined by our analysis and system-level simulation. Depending on its location region, a femtocell is self-configured to be inner femtocell or outer femtocell, which operates in dedicated-channel spectrum usage and co-channel spectrum usage, respectively. From a whole system perspective, the spectrum usage modes of femtocells are mixed in macrocell, this is why we call the proposed strategy hybrid spectrum arrangement. When looking back our proposed strategy, we realize that hybrid spectrum arrangement is a natural consequence when the femtocells can select proper spectrum usage mode by self-configuration according to their spatial conditions. 
When the proposed hybrid spectrum arrangement is employed in two-tier LTE system, the cross-tier interferences are distinct compared to that with two traditional strategies of spectrum arrangement. Specifically, the interference scenario IV (the downlink interference from eNB to HUE) and the interference scenario III (the uplink interference from MUE to HeNB) are not significant ones. The residual significant interferences, the interference scenario II (the downlink interference from HeNB to MUE) and interference scenario I (the uplink interference from HUE to eNB), can be further dealt with our proposed corresponding interference mitigation schemes.

To utilize our proposed methods in the practical twotier LTE system, we suggest to implement the hybrid spectrum arrangement along with the related interference mitigation methods as an overall solution to improve spectrum utilization. Some proposed methods in this article may not be fully compliant with the LTE standard, they could be implemented as vendor-specific solutions. After further evaluation, we expect that our proposed solution can be applied to other OFDM-based two-tier wireless system, such as WiMAX network.

\section{Competing interests}

The authors declare that they have no competing interests.

\section{Acknowledgements}

This article was supported by the National Natural Science Foundation of China (Grant No. 61062006) and the Natural Science Foundation of Hainan Province, China (Grant No. 610215).

\section{Author details}

${ }^{1}$ College of Information Science \& Technology, Hainan University, 58 Renmin Ave., Haikou, Hainan 570228, China. ${ }^{2}$ DOCOMO Beijing Communications Laboratories Co., Ltd, 7/F, Raycom Infotech Park Tower A, No. 2 Kexueyuan South Road, Haidian District, Beijing, 100190, P. R. China.

Received: 3 October 2012 Accepted: 31 January 2013 Published: 2 March 2013

\section{References}

1. R Coombs, R Steele, Introducing microcells into macrocellular networks: a case study. IEEE Trans. Commun. 47(4), 568-576 (1999)

2. LC Wang, GL Stüber, CT Lea, Architecture design, frequency planning, and performance analysis for a microcell/macrocell overlaying system. IEEE Trans. Veh. Technol. 46(4), 836-848 (1997)

3. V Chandrasekhar, JG Andrews, A Gatherer, Femtocell networks: a survey. IEEE Commun. Mag. 46(9), 59-67 (2008)

4. P Leaves, K Moessner, R Tafazolli, Dynamic spectrum allocation in composite reconfigurable wireless networks. IEEE Commun. Mag. 42(5), 72-81 (2004)

5. Y Bai, J Zhou, L Chen, in Proceedings of GLOBECOM, vol. 1. Hybrid spectrum usage for overlaying LTE macrocell and femtocell, (Honolulu, USA, 2009), pp. 1-6

6. Y Bai, J Zhou, L Liu, L Chen, H Otsuka, in Proceedings of PIMRC, vol. 2. Resource coordination and interference mitigation between macrocell and femtocell, (Cannes, France, 2008), pp. 1401-1405

7. H Claussen, Co-Channel operation of macro- and femtocells in a hierarchical cell structure. Int. J. Wirel. Inf. Netw. 15(3-4), 137-147. doi:10.1007/s10776-008-0083-8

8. V Chandrasekhar, J Andrews, Spectrum allocation in tiered cellular networks. IEEE Trans. Commun. 57(10), 3059-3068 (2009)

9. CY Oh, M Chung, H Choo, TJ Lee, in Proceedings of International Conference on Computational Science and Its Applications (ICCSA), vol. 1. A novel frequency planning for femtocells in OFDMA-based cellular networks using fractional frequency reuse, (Fukuoka, Japan, 2010), pp. 96-106

10. $\mathrm{YWu}, \mathrm{D}$ Zhang, $\mathrm{H}$ Jiang, $\mathrm{HWu}$, in Proceedings of PIMRC, vol. 1. A novel spectrum arrangement scheme for femto cell deployment in LTE macro cells, (Tokyo, Japan, 2009), pp. 6-11

11. CHM de Lima, M Bennis, M Latva-aho, in Proceedings of GLOBECOM, vol. 1. Coordination mechanisms for stand-alone femtocells in self-organizing deployments, (Houston, USA, 2011), pp. 1-6

12. I Güvenç, M Jeong, F Watanabe, H Inamura, A hybrid frequency assignment for femtocells and coverage area analysis for co-channel operation. IEEE Commun. Lett. 12, 1-3 (2008)

13. H Bharucha, H Haas, G Auer, I Cosovic, Dynamic resource partitioning for downlink femto-to-macro-cell interference avoidance. EURASIP J. Wirel. Commun. Network. 2010(Article ID143413,2010) doi:10.1155/2010/143413

14. ME Sahin, I Güvenç, M-R Jeong, H Arslan, Handling CCl and ICI in OFDMA femtocell networks through frequency scheduling. IEEE Trans. Consumer Electron. 55(4), 1936-1944 (2009)

15. H-S Jo, C Mun, J Moon, J-G Yook, Interference mitigation using uplink power control for two-tier femtocell networks. IEEE Trans. Wirel. Commun. 8(10), 4906-4910 (2009)

16. Z Shi, MC Reed, M Zhao, On uplink interference scenarios in two-tier macro and femto co-existing UMTS networks. EURASIP J. Wirel. Commun. Network. 2010 (2010). Article ID 240745

17. Z Bharucha, G Auer, TAbe, in Proceedings of WCNC, vol. 2. Downlink femto-to-macro control channel interference for LTE, (Cancun, Mexico, 2011), pp. 1259-1264

18. MS Kim, HW Je, FA Tobagi, in Proceedings of GLOBECOM, vol. 1. Cross-tier interference mitigation for two-tier OFDMA femtocell networks with limited macrocell information, (Miami, USA, 2010), pp. 1-5

19. M Ndong, T Fujii, in Proceedings of 2011 Third International, Conference on Ubiquitous and Future Networks (ICUFN), vol. 1. Interference cancellation for spectrum shared femtocell networks with macrocell information feedback, (Dalian, China, 2011), pp. 224-229

20. JH Yun, KG Shin, in Proceedings of MobiCom, vol. 1. CTRL: a self-organizing femtocell management architecture for co-channel deployment, (Chicago, USA, 2010), pp. 61-72

21. D López-Pérez, A Valcarce, Ladányi Á, Roche de la G, J Zhang, Intracell handover for interference and handover mitigation in OFDMA two-tier macrocell-femtocell networks, (2010). Article ID 142629,2010

22. JPM Torregoza, R Enkhbat, WJ Hwang, Joint power control, base station assignment, and channel assignment in cognitive femtocell networks. EURASIP J. Wirel. Commun. Network. 2010 (2010). Article ID 285714

23. Y Zhang, R Yu, W Yao, S Xie, Y Xiao, M Guizani, Home M2M networks: architectures, standards, and QoS improvement. IEEE Commun. Mag. 49(4), 44-52 (2011)

24. R Yu, Y Zhang, S Gjessing, Y Chau, S Xie, M Guizani, Cognitive radio based hierarchical communications infrastructure for smart grid. IEEE Netw. Mag. 25(5), 6-14 (2011)

25. S Xie, Y Liu, Y Zhang, R Yu, A parallel cooperative spectrum sensing in cognitive radio networks. IEEE Trans. Veh. Technol. 59(8), 4079-4092 (2010)

26. 3GPP TR 25.820 v8.2.0, 3G Home NodeB Study Item Technical Report (2008)

27. CU Castellanos, DL Villa, C Rosa, KI Pedersen, FD Calabrese, PH Michaelsen, J Michel, in Proceedings of VTC 2008 Spring, vol. 3. Performance of uplink fractional power control in UTRAN LTE, (Marina Bay, Singapore, 2008), pp. 2517-2521

28. 3GPP TR 36.942 v10.2.0, Evolved Universal Terrestrial Radio Access (E-UTRA); Radio Frequency (RF) system scenarios (2010)

29. 3GPP TR 36.814 v9.0.0, Further Advancements for E-UTRA Physical Layer Aspects (2010)

doi:10.1186/1687-1499-2013-56

Cite this article as: Bai and Chen: Hybrid spectrum arrangement and interference mitigation for coexistence between LTE macrocellular and femtocell networks. EURASIP Journal on Wireless Communications and Networking 2013 2013:56. 